\title{
A small current grounding fault line selection based on Improved EMD
}

\author{
algorithm and Fractal Theory \\ Gao Xuehan ${ }^{1}$ \\ ${ }^{1}$ School of Northeast Petroleum University, Daqing, 163318, China \\ 1393796776@qq.com.
}

Keywords: small current grounding system; fault line selection; empirical mode decomposition; fractal theory; correlation dimension

\begin{abstract}
This paper systematically analyzes the small current grounding system grounding and the principle of the failure formation, combined with empirical mode decomposition, and then put forward a new method of empirical mode decomposition and correlation dimension for small current grounding system of single phase to ground fault line selection. Finally, by MATLAB data processing, through the zero-sequence current empirical mode decomposition and extracts harmonic components after calculating the correlation dimension. It affects the factors of the small current grounding system fault line selection of single-phase ground, and tests the fault line method and criterion reliability.
\end{abstract}

\section{Introduction}

At present, China $35 \mathrm{kV}$ and below voltage level, it widely used neutral point arc suppression coil grounding, by compensating for the inductor current to reduce ground current, reduce the line fault trip rate. Since the single-phase ground fault accounted for more than $80 \%$ of the distribution network fault, it is especially important to accurately select the fault line when single-phase ground fault occurs. Relatively new morphological filtering algorithms, owing to the sensitive ground fault angle is limited in practical use. The results show that the improved EMD algorithm combined with the fractal theory can effectively solve the problem of end effect, thereby improving the accuracy of single-phase ground fault line selection.

\section{Improved EMD Algorithm}

Improved EMD Algorithm. This article will use LSSVM for signal extension to suppress the end effect. Using LSSVM continuation results are better, but time-consuming. And if the signal is a monotonous signal it will never appear extreme point. This is the most common problem that based on same sampling interval extension.

For a sampling signal

$$
s=(1+\sin (20 \pi t)) \cdot \sin (160 \pi t)+\sin (300 \pi t), t \in[0,0.2]
$$

To the original signal which has not undergone any treatment conducted EMD. Obtained as shown in (1) shown a series of IMF. From Figure 1 shows that each IMF component at the breakpoint diverges very serious. And further transmits to the internal sequence, so that each component of the IMF at the endpoints is serious distortion. Curve almost coincide, end effect is greatly reduced. EMD energy leakage after LSSVM process will be less.

Mode mixing. In the signal after EMD decomposition will appear in a single IMF contains a 
different time scale. This situation is known as Mixing Mode. The mode mixing appears to be related to the EMD algorithm, and is influenced by the frequency and amplitude of the signal. The results show that the relationship between the frequency component and the amplitude of the signal affects the mode mixing phenomenon when the EMD signal is decomposed.

In view of the common frequency spread mode aliasing in power system, the sine signal $250 \mathrm{~Hz}$ and $50 \mathrm{~Hz}$ of $\mathrm{x}(\mathrm{t})$ as an example:

$$
x(t)=6 \sin (2 \pi \times 50 t)+\sin (2 \pi \times 250 t)
$$

According to our understanding of and empirical mode decomposition (EMD) shall appear such two IMF components, the first IMF component frequency is $250 \mathrm{~Hz}$, the second the IMF should be $50 \mathrm{~Hz}$ frequency component, which is based on this conjecture, we signal $\mathrm{x}(\mathrm{t})$ of EMD decomposition, the decomposition results as shown in the figure 1 .
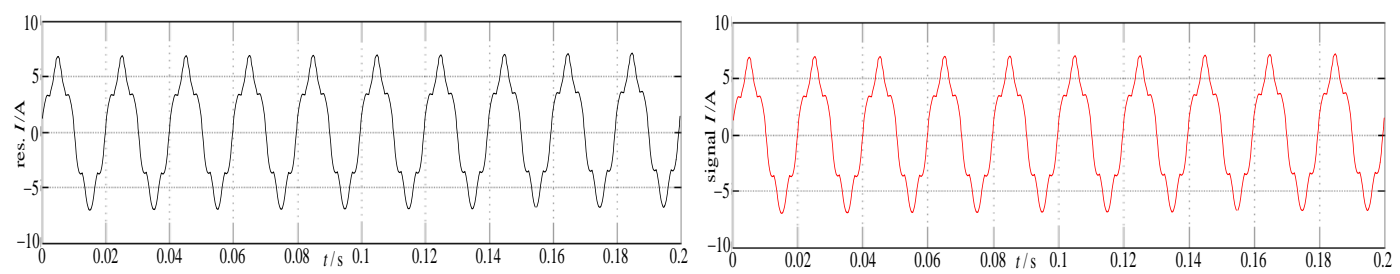

Fig.1 EMD decomposition results chart of signal $x(t)$
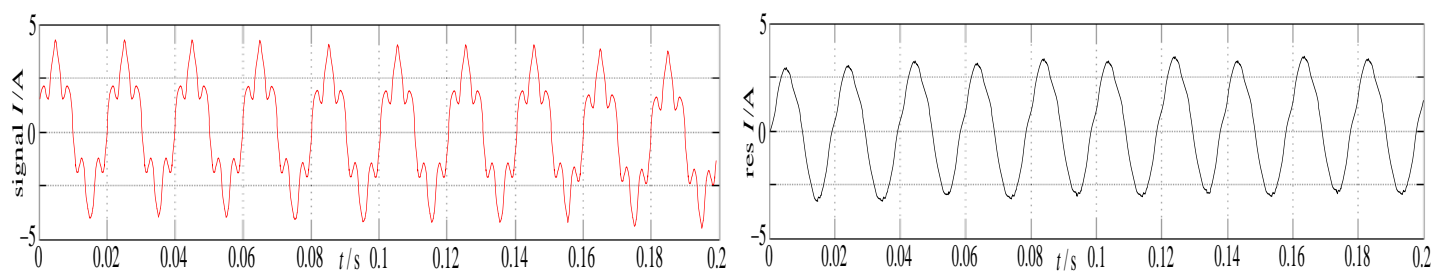

Fig.2 EMD decomposition results after adjusting the amplitude

According to their inference, we change the signal amplitude ratio of $50 \mathrm{~Hz}$ and $250 \mathrm{~Hz}$, so that it can meet the conditions that they do not occur mode mixing:

$$
x(t)=3 \sin (2 \pi \times 50 t)+\sin (2 \pi \times 250 t)
$$

EMD decomposition of the signal, as shown in figure 2 .

Can be seen from the figure, after the adjustment of the amplitude, EMD decomposition will be effective to out of the two frequency points. Effective solution to the problem of mode mixing.

\section{Correlation dimension fault line selection method}

Correlation dimension is one of the common dimension. Correlation Dimension can reflect the characteristics of the system in a different states, the states in which the distinction between systems based on different dimension. The practical application is using G-P algorithm to calculate the correlation dimension.

Introduction Algorithm. G-P algorithm is embedding space by observing the radius $\mathrm{r}$, decreases with the radius $\mathrm{r}$, G-P algorithm is by looking at embedding space radius $r$, with the radius $r$ decreases, the number of points in space is gradually reduced to zero in the process to calculate the correlation dimension.

Method of fault line selection. Fault transient waveform and fault angle of single-phase ground are 
closely linked. High frequency transient component failure is not obvious, the first wave direction of the fault determination by the fault line.

\section{Simulation verification}

Simulation model. In order to verify the feasibility of the proposed fault line selection scheme, the simulation model of single phase to ground fault in $10 \mathrm{kv}$ distribution network is established which is based on Matlab/Simulink simulation software. Distribution network by eliminating arc suppression coil compensation and the compensation degree is $8 \%$, the simulation of the cables distribution parameters $\mathrm{L}_{0}=0.398 \mathrm{mH} / \mathrm{km}, \mathrm{C}_{0}=0.203 \mu \mathrm{F} / \mathrm{km}$.. Simulation outlet 3 distance bus $2 \mathrm{~km}$ single-phase grounding fault occurs.

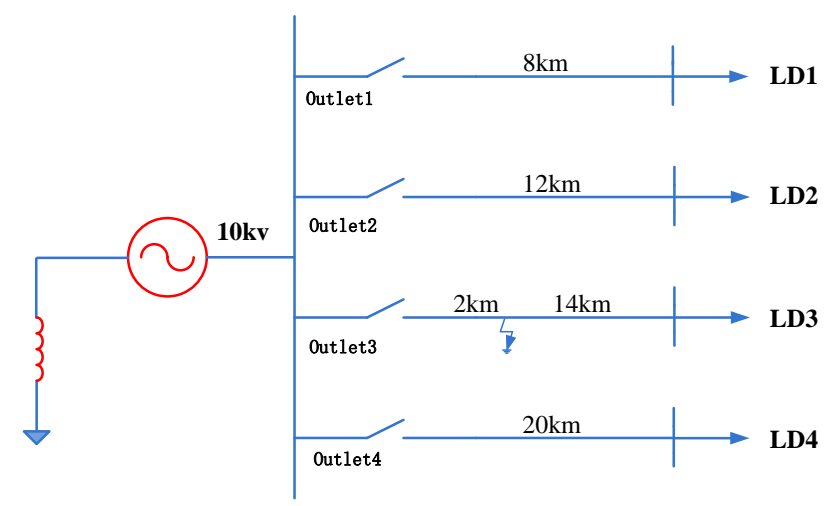

Fig.3 Simulation of single-phase earth fault

Result analysis. Collecting the line fault of a power zero mode current, before semi period of the mains frequency and after semi period of the mains frequency, the wire current improved empirical mode decomposition (EMD). Setting angle of less than $15^{\circ}$ the fault time waveform monotonically of discrimination, so according to the requirements to get the fault angle for $0^{\circ}$ each outlet fault waveform and the Improved EMD algorithm to filter out the high frequency components of low-frequency fault waveform, as shown in the figure 4 .
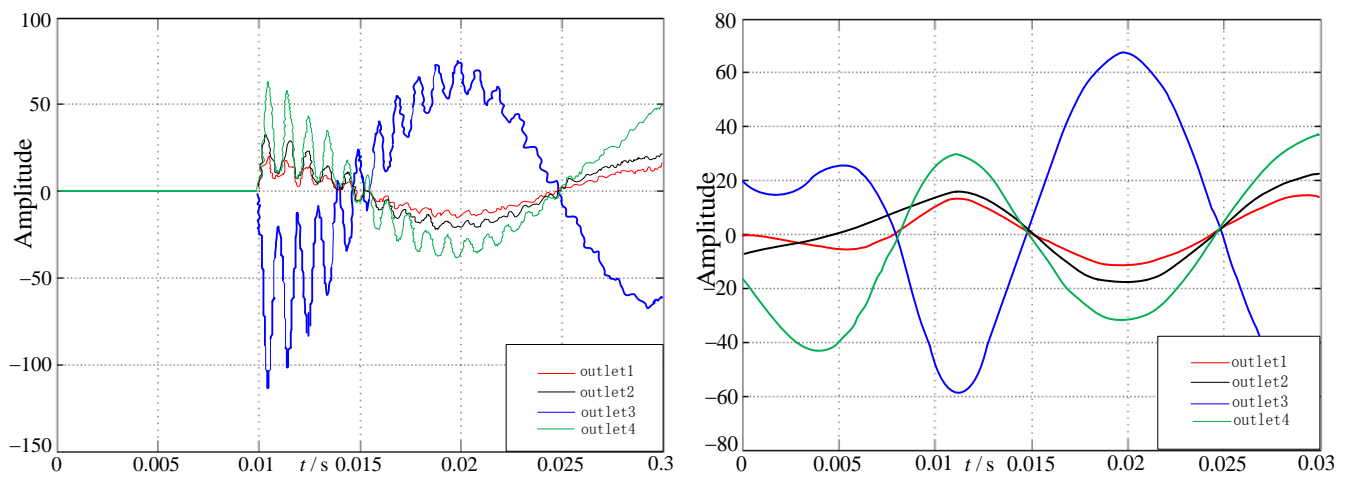

(a)Outlet wave

(b)Low frequency fault waveform

Fig.4 Waveforms of the line and low frequency under the fault angle of $0^{\circ}$

From the graph shows that in the vicinity of the $0.01 \mathrm{~s}$ and the failure time, the outlet 3 is monotone decreasing, while the other outlet is increasing, the fault line and the non fault line is the opposite. Simulation results show that the high frequency interference current of $5100 \mathrm{~Hz}$ in IMF1. Shown in figure $30^{\circ}$ fault angle outlet 3 fault feature waveform EMD algorithm and Improved EMD algorithm comparison, as can be seen, the improved EMD algorithm basically filter out the high frequency interference current. 

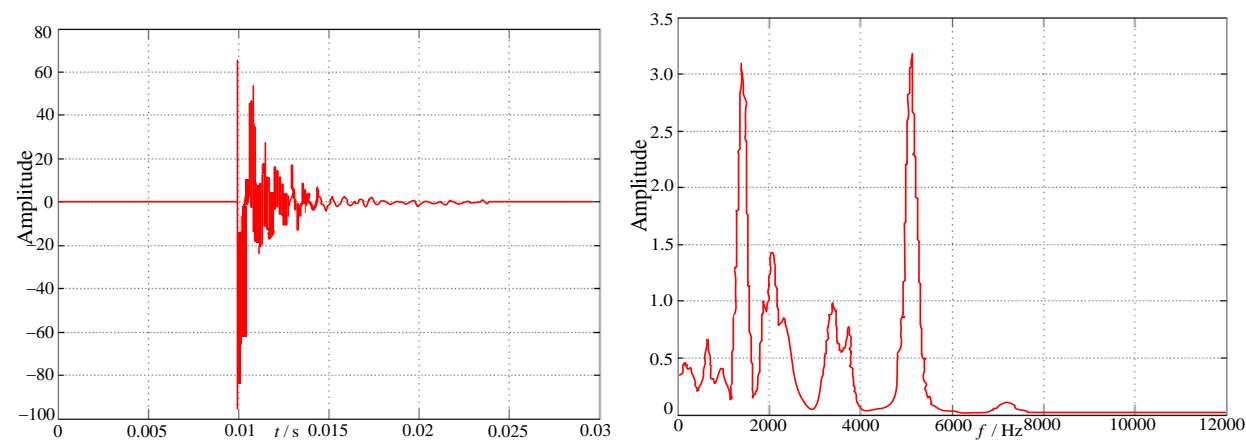

(a) Fault characteristic waveform and FFT analysis by EMD decomposition
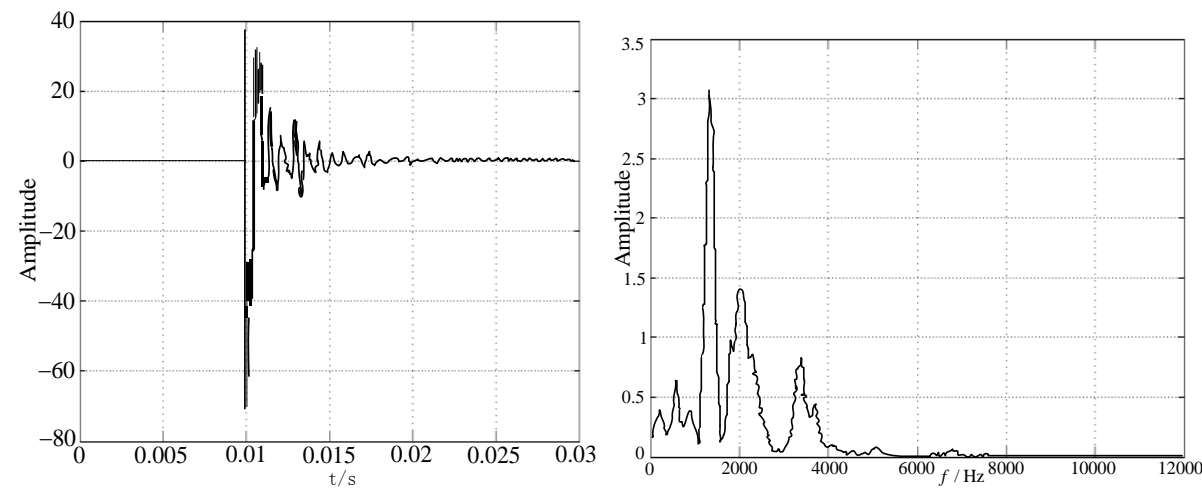

(b)Fault characteristic waveform and FFT analysis of improved EMD decomposition

Fig.5 The line 3 waveform of fault characteristic waveform under the angle of $30^{\circ}$

\section{Summary}

This paper analyzes and proposes a method to identify the fault line by using the improved EMD algorithm. Based on the analysis of the fault transient process of the small current grounding system, it can identify system characteristic frequency bands. The improved EMD algorithm proposed in this paper can avoid the possibility of the error caused by the high frequency interference, and improve the accuracy of the line selection. The simulation results show the proposed method is feasible to improve the line selection, line selection for distribution automation provides a feasible scheme.

\section{References}

[1] PHADKE A G. Synchronized Phasor Measurements-A Historical Overview. In: Proceedings of IEEE/PES Transmission and Distribution Conference and Exhibition 2002, Asia Pacific, Vol 1, Yokahama(Japan): 2002,476-479.

[2] PHADKE A G. Synchronized Phasor Measurements in power Systems. IEEE Computer Applications in Power,1993, 6(2): 10-15.

[3] IEEE Standard 1344-1995(R2001). IEEE Standard for Synchrophasors for Power Systems.

[4] Zhang Yuhui, Wang Lijun, Lan Hua, et al. A method of resonant earthed system fault line detection based on EMD[J]. Electrical Measurement Instrumentation, 2012, 49(12): 7-11.

[5] Huang N E, Zheng S, Steven R, et al. The empirical mode decomposition and Hilbert spectrum for nonlinear and nonstationary time series analysis[J]. Proceedings of the Royal Society of London, 1998, 454: 903-995.

[6] Shu Hongchun, Zhao Wenyuan, Pen Shixin. Faulty line selection based on HHT detection for hybrid distribution network[J]. Electric Power Automation Equipment, 2009, 29(5): 4-9. 